\title{
KERNEL-BASED UNSUPERVISED CHANGE DETECTION OF AGRICULTURAL LANDS USING MULTI-TEMPORAL POLARIMETRIC SAR DATA
}

\author{
M. A. Fazel ${ }^{\text {a, }}$, S. Homayouni ${ }^{\text {b }}$, J. Amini $^{\text {a }}$ \\ ${ }^{\mathrm{a}}$ Dept. of Geomatics Engineering, College of Engineering, University of Tehran, Iran - (m.a.fazel, jamini)@ut.ac.ir
${ }^{\mathrm{b}}$ Dept. of Geography, University of Ottawa, Canada - saeid.homayouni@uOttawa.ca
}

KEY WORDS: Change Detection, Synthetic Aperture Radar, Polarimetric SAR, Agricultural lands monitoring, Kernel-based cmeans, multi-temporal data analysis.

\begin{abstract}
:
Unsupervised change detection of agricultural lands in seasonal and annual periods is necessary for farming activities and yield estimation. Polarimetric Synthetic Aperture Radar (PolSAR) data due to their special characteristics are a powerful source to study temporal behaviour of land cover types. PolSAR data allows building up the powerful observations sensitive to the shape, orientation and dielectric properties of scatterers and allows the development of physical models for identification and separation of scattering mechanisms occurring inside the same region of observed lands. In this paper an unsupervised kernel-based method is introduced for agricultural change detection by PolSAR data. This method works by transforming data into higher dimensional space by kernel functions and clustering them in this space. Kernel based c-means clustering algorithm is employed to separate the changes classes from the no-changes. This method is a non-linear algorithm which considers the contextual information of observations. Using the kernel functions helps to make the non-linear features more separable in a linear space. In addition, use of eigenvectors' parameters as a polarimetric target decomposition technique helps us to consider and benefit physical properties of targets in the PolSAR change detection. Using kernel based c-means clustering with proper initialization of the algorithm makes this approach lead to great results in change detection paradigm.
\end{abstract}

\section{INTRODUCTION}

Polarimetric Synthetic Aperture Radar (PolSAR) data provide high resolution, weather-independent, day-and-night images for various remote sensing applications such as land cover change detection. These types of data allows to build up the powerful observations sensitive to shape, orientation and dielectric properties of the scatterers and allows the development of physical models for identification and separation of scattering mechanisms occurring inside the same region of observed land cover. In addition, monitoring of agricultural lands requires the potential data to study temporal behavior of land in seasonal and annual periods for farming activities and yield estimation (Coppin and Bauer 1996).

Change detection methods are generally grouped into supervised and unsupervised methods. Supervised methods work based on multi-temporal land-cover mapping of satellite images in which the accuracy of detected changes highly depends on the classification accuracy (Lu et al. 2004). For real applications, collecting of ground reference information is normally expensive and time consuming. On the other hand, the unsupervised methods extract the information of images without any a priori knowledge information (Bruzzone and Prieto 2000). These methods can be applied to either pixel or feature vectors, which are obtained directly from images.

Several unsupervised change detection techniques including the very simple idea of image differencing and Change Vector Analysis (CVA) to more sophisticated statistical modeling of changes in images have been reviewed in literature (Radke et al. 2005). For SAR change detection, generalized Gaussian model method (Bazi, Bruzzone, and Melgani 2005) and generalized minimum-error threshold methods (Moser and Serpico 2006) have been proposed. An algorithm based on Markov Random Field model (MRF) is used in (Kasetkasem and Varshney 2002) to find the optimum image of change and no-change classes. Bovolo et al. (2008) proposed an algorithm based on the Support Vector Machines (SVM) as unsupervised change detection algorithm which uses the binary classification of change and no-change classes (Bovolo, Bruzzone, and Marconcini 2008).

In recent years, the use of kernel-based methods in change detection applications has become an interesting topic in remote sensing community. Kernel-based methods and machine learning algorithms are the unsupervised paradigms which introduced powerful tools to deal with nonlinear classification (Camps-Valls and Bruzzone 2009). Kernel methods allow performing a linear analysis into a higher dimensional feature space, corresponding to a nonlinear solution in the original input space.

In this paper, we present an unsupervised framework for detecting the changes of agricultural lands. The proposed change detection method uses the kernel-based clustering technique. Kernel c-means algorithm is employed to separate the changes from no-change classes of images. This method is a non-linear algorithm which considers the contextual information of the images. To benefit the polarimetric characteristics of PolSAR data, we used H-A- $\alpha$ decomposition (or Cloude-Pottier) which is derived from the eigenvectors and the eigenvalues of coherency matrix of original scattering matrix observation. This decomposition has well adaption for agricultural applications and can help us to separate different scattering mechanisms of agricultural lands. In addition, we

\footnotetext{
Corresponding author.
} 
applied our method to single channel images as well and the results are compared.

The proposed method consists of a multi-step framework. In the first step, change maps are calculated using the multi-temporal images, i.e. one image per paired-date. Then, these change images are projected into a higher dimensional space, using the kernel functions. The projection to higher dimensional space helps to make the non-linear separable features linearly separable. Because of non-linear behavior of changed phenomenon, applying the non-linear version of change detection algorithms seem to lead to more reliable results. The final step is using an unsupervised technique to obtain the change and no-change classes and for this purpose, the clustering algorithm is employed.

We have applied the proposed methodology to several full polrimetric L-band SAR image datasets acquired by the Uninhabited Aerial Vehicle Synthetic Aperture Radar (UAVSAR) over an agricultural area in Winnipeg, Canada. This area consists of a rich variety of annual crops including cereals, wheat, oilseeds, soybeans, corn, and pasture/forage. The obtained time series change maps can be used to evaluate and assess the behavior of agricultural lands. In the rest part of this paper, several kernels are introduced based on using that take into account the spectral, spatial and temporal information for change detection approach. Then the basic formulation of our proposed method is presented and finally the practical implemented results and discussion conclude the paper.

\section{METHODOLOGY}

\subsection{Kernel Principals}

Generally, the kernel functions are applied to overcome the problems of explicit mapping function's computation. In the feature space, using a kernel means that the value of dot product is directly evaluated by using the value of the samples in input space. Transformed samples are more likely linearly separable in the resulting feature space (Camps-Valls et al. 2008). The higher dimensional space is induced by a mapping function $\phi$, which $\phi(x)$ is mapped sample in higher space $H$. The kernel values show the similarity between samples. Some popular kernels are: linear, polynomial and radial basis function (RBF) (Camps-Valls and Bruzzone 2009).

For change detection purpose, the kernel function can be applied onto the change difference images. Then, in addition to mentioned kernels, Difference Kernel can be obtained by subtracted maps in the higher dimension space. In higher dimension space, the differences of same sample in two subsequent images are defined as:

$$
\begin{aligned}
& \phi\left(\mathrm{x}_{\mathrm{i}}\right)=\phi\left(x_{i}^{\left(t_{1}\right)}\right)-\phi\left(x_{i}^{\left(t_{1}\right)}\right)= \\
& \phi_{1}\left(x_{i}^{(1)}\right)-\phi_{2}\left(x_{i}^{(2)}\right), \phi_{2}\left(x_{i}^{(2)}\right)-\phi_{3}\left(x_{i}^{(3)}\right) \\
& , \ldots, \phi_{T-1}\left(x_{i}^{(T-1)}\right)-\phi_{T}\left(x_{i}^{(T)}\right)
\end{aligned}
$$

And by replacing the corresponding dot product with a proper kernel function $K$, the kernel function equations can be easily computed as equation (2).

$$
\left.K\left(x_{i}, x_{j}\right)=\sum_{t=1}^{T-1}\left(K_{t}\left(x_{i}^{t}, x_{i}^{t}\right)+K_{i}^{t}, x_{j}^{t+1}\right)-K_{t}\left(x_{j}^{t+1}, x_{j}^{t+1}, x_{j}^{t}\right)\right)-
$$

For simplify the computation and separating single- and crosstime components we can reach equation (3) as:

$$
\begin{aligned}
& K D(x, x)=k_{t 2}\left(x_{i}^{t 2}, x_{j}^{t 2}\right)+k_{t 1}\left(x_{i}^{t 1}, x_{j}^{t 1}\right)- \\
& k_{t 2 t 1}\left(x_{i}^{t 2}, x_{j}^{t 1}\right)-k_{t 1 t 2}\left(x_{i}^{t 1}, x_{j}^{t 2}\right)
\end{aligned}
$$

where $\quad \mathrm{t} 1$ and $\mathrm{t} 2$ show first and last time,

$k_{t 2 t 1}$ and $k_{t 1 t 2}$ are cross-time kernel functions.

Then the resulting difference kernel is composed of four kernels accounting for single time and cross-time similarity information between images (Camps-Valls et al. 2008).

\subsection{Kernel-based C-means for Change Detection}

The kernel c-means algorithm is a kernelized version of the well-known c-means clustering technique. In other words, cmeans is particularly performed to solve the linear problems where the input space is organized in spherical or radial clusters. The kernel version of c-means instead of working in real physical input space $X$, works in a higher dimensional feature space $H$. By this dimensionally transform, the clusters which are non-spherical in input space, are mapped into a spherical one, and consequently can be clustered easily and correctly (Camps-Valls and Bruzzone 2009).

The classical c-means approach is useful to aim a natural partitioning of the input pattern $X$ into $c$ groups. The algorithm works by minimizing distance of elements $x_{i}$ from cluster's gravity center $m_{c}$ and assigns a cluster membership $c$ to the elements (Kanungo et al. 2002). The algorithm uses an iterative refinement technique. When the pattern assigned to the corresponding clusters, the mean vector $m_{c}$ is updated by averaging the coordinates of elements of the cluster. Then it provides a new gravity center. The process is iterated until the centers became consistence and algorithm reaches to a minimum of $d^{2}\left(x_{i}, m_{c}\right)$ (Kanungo et al. 2002). By considering the mapping function $\phi$ in kernel space, the similarity measure of cmeans becomes as equation (4):

$$
d^{2}\left(\varphi\left(x_{i}\right), m_{c}\right)=\left\|\varphi\left(x_{i}\right)-m_{c}\right\|^{2}, m_{c}=\frac{1}{\left|N_{c}\right|} \sum_{j \in C_{c}} \varphi\left(x_{i}\right)
$$

Then kernel c-means method first groups similar points in higher dimension and then separate them (Camps-Valls and Bruzzone 2009). By expansion of the equation (4) and replacing dot product by a proper kernel function, the kernel c-means formulation is obtained as:

$$
\begin{aligned}
& d^{2}\left(\varphi\left(x_{i}\right), m_{c}\right)=\left\langle\varphi\left(x_{i}\right), \varphi\left(x_{i}\right)\right\rangle+ \\
& \frac{1}{\left|N_{c}\right|^{2}} \sum_{j, m \in C_{c}}\left\langle\varphi\left(x_{j}\right), \varphi\left(x_{m}\right)\right\rangle-\frac{2}{\left|N_{c}\right|} \sum_{j \in C_{c}}\left\langle\varphi\left(x_{i}\right), \varphi\left(x_{j}\right)\right\rangle= \\
& k\left(x_{i}, x_{i}\right)+\frac{1}{\left|N_{c}\right|^{2}} \sum_{j, m \in C_{c}} k\left(x_{j}, x_{m}\right)-\frac{2}{\left|N_{c}\right|} \sum_{j \in C_{c}} k\left(x_{i}, x_{j}\right)
\end{aligned}
$$


The main considerations of proposed kernel based c-means CD algorithm are initialization and optimization of the cost function. It is obvious that one of the limitations of iterative techniques is the initialization issue. Poor initialization can converge in the local minima, and then the algorithm fails (Kanungo et al. 2002). In order to reach a stable and correct grouping, one possible solution is to initialize the algorithm with a training set which are based on the prior analysis.

\subsection{Initialization}

First step of our proposed method for unsupervised change detection algorithm of PolSAR data is the initialization. Figure 1 illustrates the first step consideration thematically.

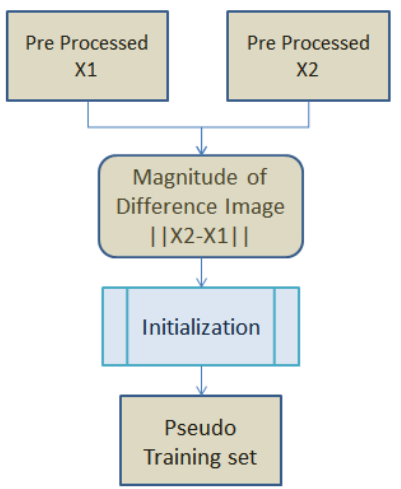

Figure 1. Figure placement and numbering

In this paper, the method presented in (Bruzzone and Prieto 2000) is used for initialization purpose. The magnitude of the difference image is used to estimate the mean and standard deviation of a mixture of two Gaussian distributions. This hypothesis and threshold is used to initialize the algorithm genuinely. Figure 2 shows thematically the overlap area of two distributions. Then the samples far from overlap zone are used to training of the algorithm.

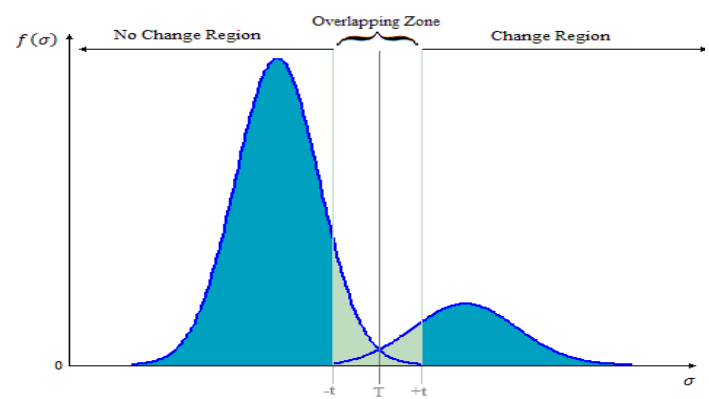

Figure 2. Mixture of two Gaussians histograms for change and no-change area.

Pixels with a magnitude close to zero correspond to unchanged region and pixels corresponding to values far from zero are assumed as changes in the state of surfaces. Then, a threshold proportional to the standard deviations is defined and the training samples are randomly extracted from the regions that are labeled as changed and un-changed in the histogram.

To estimate the cluster centers, the kernel c-means (5) is used to partition the training samples. However, different kernel functions can be used in this stage; we use the RBF and Difference Kernels.

\subsection{Kernel Parameter Estimation}

RBF kernel related to Gaussian distribution and for this reason have adaptable results. On the other hand, the changes associated to differences between two images and difference kernel contains admissible information about changes (Kim et al. 2005). We used RBF kernels for the kernels generate difference kernel (2). Then an optimization problem which is the distance between samples and their cluster center, must be solved.

To obtain the difference kernel's parameters, two single- and cross-time parameters should be estimated (Kim et al. 2005). The Rayleigh coefficient, in term of cluster-related distance in feature space, is presented in equation (6). This coefficient is calculated from the covariance or normalized scatter matrices of data (Kim et al. 2005).

$$
\left\{\sigma^{\text {sin } g l e}, \sigma^{\text {cross }}\right\}=\arg \min \left\{\frac{\sum_{c} \frac{1}{m_{c}} \sum_{i \in c} d^{2}\left(\varphi^{d i f f}\left(x_{i}\right), m_{c}\right)}{\sum_{c \neq q} d^{2}\left(m_{c}, m_{q}\right)}\right\}
$$

It's worth noting that in order to minimize this expression, the kernel c-means technique is wrapped to test different sets of parameters to cluster the same training samples. For this purpose, we used linear search method to obtain the best parameters.

After initialization and estimation of optimum kernel parameters, we can apply optimal kernel c-means algorithm on our data sets. Figure 3 presents the flowchart of main step for our approach which is used in this research.

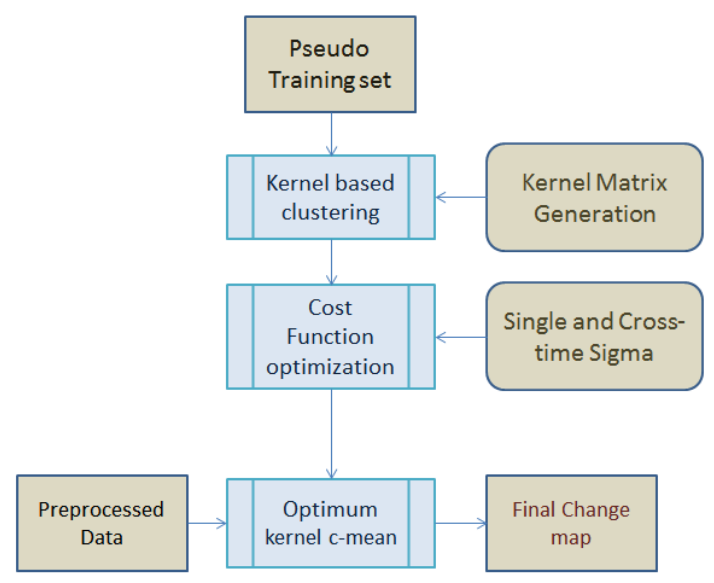

Figure 3. Overview for the main step of the proposed approach.

Several features derived from PolSAR data sets can be used in change detection algorithm. Cloude-Pottier decomposition which is derived from the eigenvectors and the eigenvalues of coherency or covariance matrix observation has well adaption for agricultural applications and can help us to separate different scattering mechanisms of agricultural lands. In addition, we applied our method to single channel images as well and the results are compared in term of quantitative metrics which are presented in the next section. 


\section{RESULTS AND DISCUSSION}

Our approach starts with a preprocessing step for speckle modeling and reduction from SAR images, and radiometric correction. Then co-registration of the images and selecting of common scene in several images is necessary. In the second step, the change maps are calculated using the multi-temporal images, i.e. one map per paired-date. Then, these change images are projected to a higher dimensional space using the kernel functions. The final step is using an unsupervised technique to obtain the change and no-change classes. For this purpose, the kernel-based c-means clustering algorithm is employed.

The proposed algorithm has applied to two datasets of L-band PolSAR data sets, acquired by the UAVSAR over an agricultural area in Winnipeg, Canada. Figure 4 shows a $2351 \times 2432$ subsample of pauli decomposition of data which is used in this research.

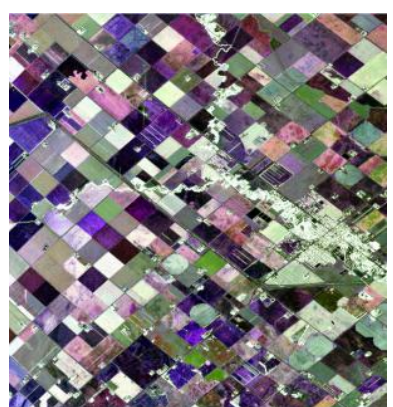

(a)

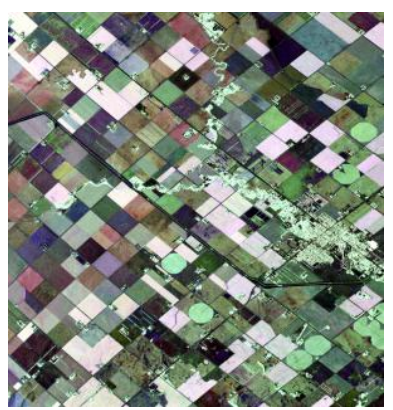

(b)
Figure 4. Pauli decomposition of sub-sample images acquired in (a) 17 Jun and (b) 17 July 2012.

This area consists of a rich variety of annual crops including cereals, wheat, oilseeds, soybeans, corn, and pasture/forage. The images have been acquired in 17 June and July 2012 and are in multi look stokes matrix format. The algorithm is implemented on a co-registered pre-processed data.

Our approach is principally applied on stack images which are produced by Cloude-Pottier decomposition parameters. These parameters i.e. alpha, anisotropy and entropy are shown in figure 5 .

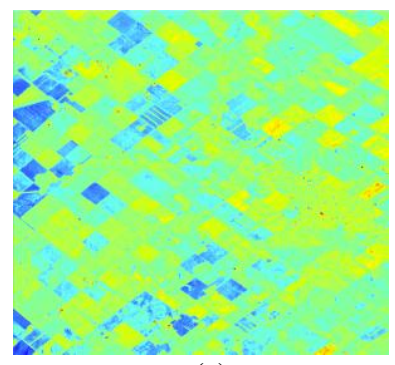

(a)

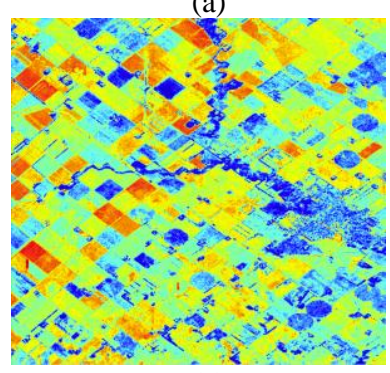

(c)

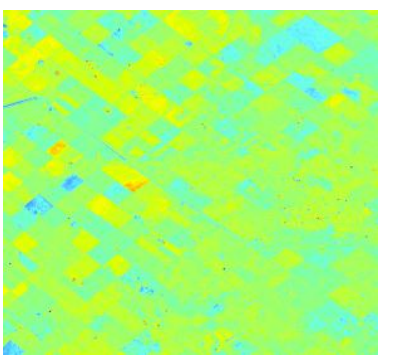

(b)

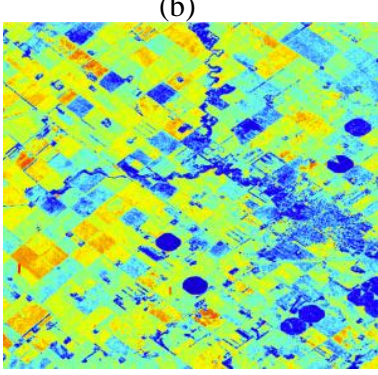

(d)

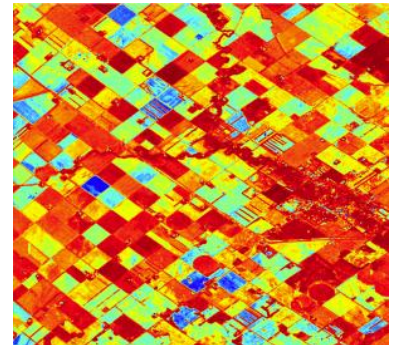

(e)

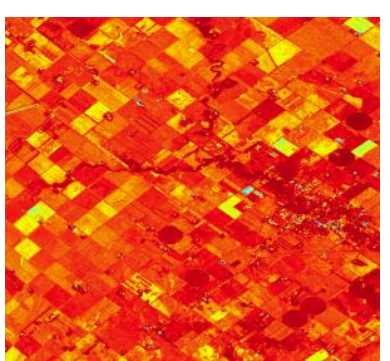

(f)
Figure 5. H-A- $\alpha$ decomposition parameters, $(\mathrm{a}, \mathrm{b})$ alpha, $(\mathrm{c}, \mathrm{d})$ anisotropy and (e,f) entropy for Jun and July respectively.

The results of difference kernel in c-means clustering based change detection with $\mathrm{H}-\mathrm{A}-\alpha$ stack images are showed in Fig 6 . The stack image is used to consider all parameters of $\mathrm{H}-\mathrm{A}-\alpha$ decomposition in change detection approach.

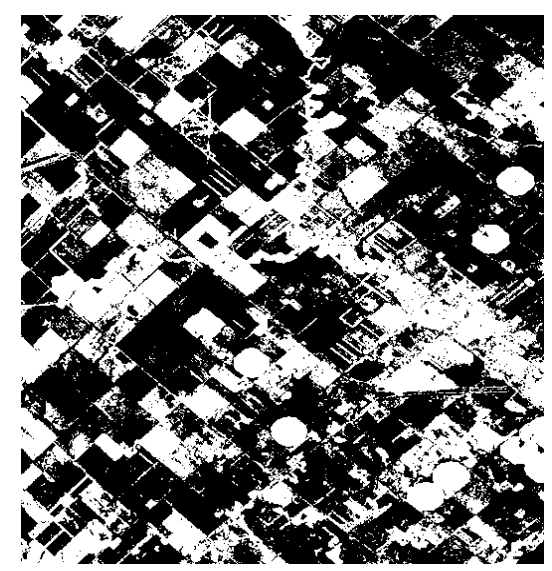

Figure 6. Resulted change map by proposed method. Black colour described as change and white show un-changed classes.

Difference of entropy and anisotropy in the temporal dataset is the reason of large amount of detected changes. For identification of detected changes using auxiliary data such as soil moisture and vegetation height can be very interesting.

For the quantitative validation of presented approach, 216 pixels are used to evaluate the confusion matrices, kappa coefficient and overall accuracy. These data have been uniformly selected from the change and no-change classes. Also the results are compared with the detected changes by classic cmeans clustering algorithm in the term of quantitative parameters. Table 1 shows quantitative comparison between the results of our approach and the c-means clustering method and also single channel images for change detection paradigm.

\begin{tabular}{|c|c|c|c|c|}
\hline $\begin{array}{c}\text { Method/ } \\
\text { Accuracy }\end{array}$ & $\begin{array}{c}\text { False } \\
\text { Alarm (\%) }\end{array}$ & $\begin{array}{c}\text { Misdetection } \\
(\%)\end{array}$ & Kappa & $\begin{array}{c}\text { OA } \\
(\%)\end{array}$ \\
\hline $\begin{array}{c}\text { c-means } \\
\text { clustering }\end{array}$ & 15.69 & 6.52 & 0.7779 & 88.89 \\
\hline $\begin{array}{c}\text { KCMCD } \\
\text { (single ch.) }\end{array}$ & 9.28 & 4.38 & 0.8634 & 93.17 \\
\hline $\begin{array}{c}\text { KCMCD } \\
\text { (RBF) }\end{array}$ & 7.46 & 3.71 & 0.8883 & 94.41 \\
\hline $\begin{array}{c}\text { KCMCD } \\
\text { (DK) }\end{array}$ & 5.77 & 1.95 & 0.9228 & 96.14 \\
\hline
\end{tabular}

Table 1. Quantitative comparison of the results. KCMCD is abbreviation to kernel based c-means change detection. 
In this table, the false alarm rate and misdetection rate consequently show the detected changes which are not actual changes and the un-changed pixels which the algorithm considers them as changed pixels. These elements of confusion matrices and also total accuracy and kappa coefficient show improvement of the kernel-based algorithm in accurate detection of changes. Moreover the use of $\mathrm{H}-\mathrm{A}-\alpha$ decomposition instead of single channel images leads to better outcome.

\section{CONCLUSION}

In this paper a kernel-based change detection algorithm is applied on full polarimetric SAR images. By transforming preprocessed co-registered images into kernel space and separating change and un-change classes by c-means algorithm, resulted change maps are obtained. Non-linear version change detection leads to more reliable results. Using RBF kernel helps to extract non-spherical classes of change correctly and moreover difference kernel reach more adaptive results in change detection applications. The quantitative comparison emphasizes the efficiency of the proposed approach. The obtained time series change maps can be used to evaluate and assess the environmental behavior of agricultural lands especially.

\section{ACKNOWLEDGMENT}

The authors acknowledge NASA/JPL-Caltech for UAVSAR data over the SMAPVEX12 area in Winnipeg, MN, Canada, and Dr. H. McNairn from Agriculture and Agri-Food Canada for her helpful support and useful discussion.

\section{References}

Bazi, Y., L. Bruzzone, and F. Melgani. 2005. “An Unsupervised Approach Based on the Generalized Gaussian Model to Automatic Change Detection in Multitemporal SAR Images." Geoscience and Remote Sensing, IEEE Transactions On 43 (4): 874-887.

Bovolo, F., L. Bruzzone, and M. Marconcini. 2008. "A Novel Approach to Unsupervised Change Detection Based on a Semisupervised SVM and a Similarity Measure." Geoscience and Remote Sensing, IEEE Transactions On 46 (7): 2070-2082.

Bruzzone, L., and D. F. Prieto. 2000. "Automatic Analysis of the Difference Image for Unsupervised Change Detection." Geoscience and Remote Sensing, IEEE Transactions On 38 (3): 1171-1182.

Camps-Valls, G., and L. Bruzzone. 2009. Kernel Methods for Remote Sensing Data Analysis. Wiley Online Library. http://onlinelibrary.wiley.com/doi/10.1002/97804707 48992.fmatter/summary.

Camps-Valls, G., L. Gómez-Chova, J. Muñoz-Marí, J. L. RojoAlvarez, and M. Martínez-Ramón. 2008. "Kernelbased Framework for Multitemporal and Multisource Remote Sensing Data Classification and Change Detection." Geoscience and Remote Sensing, IEEE Transactions On 46 (6): 1822-1835.

Coppin, Pol R., and Marvin E. Bauer. 1996. "Digital Change Detection in Forest Ecosystems with Remote Sensing Imagery." Remote Sensing Reviews 13 (3-4): 207234.
Kanungo, T., D. M. Mount, N. S. Netanyahu, C. D. Piatko, R. Silverman, and A. Y. Wu. 2002. "An Efficient Kmeans Clustering Algorithm: Analysis and Implementation." Pattern Analysis and Machine Intelligence, IEEE Transactions On 24 (7): 881-892.

Kasetkasem, T., and P. K. Varshney. 2002. "An Image Change Detection Algorithm Based on Markov Random Field Models." Geoscience and Remote Sensing, IEEE Transactions On 40 (8): 1815-1823.

Kim, Dae-Won, Ki Young Lee, Doheon Lee, and Kwang H. Lee. 2005. "Evaluation of the Performance of Clustering Algorithms in Kernel-induced Feature Space." Pattern Recognition 38 (4): 607-611.

Lu, D., P. Mausel, E. Brondizio, and E. Moran. 2004. "Change Detection Techniques." International Journal of Remote Sensing 25 (12): 2365-2401.

Moser, G., and S. B. Serpico. 2006. "Generalized Minimumerror Thresholding for Unsupervised Change Detection from SAR Amplitude Imagery." Geoscience and Remote Sensing, IEEE Transactions On 44 (10): 2972-2982.

Radke, R. J., S. Andra, O. Al-Kofahi, and B. Roysam. 2005. "Image Change Detection Algorithms: a Systematic Survey." Image Processing, IEEE Transactions On 14 (3): 294-307. 